Meta

Journal des traducteurs

Translators' Journal

\title{
Une matrice terminologique universelle : la brachygraphie gigogne
}

\section{André Clas}

Volume 32, numéro 3, septembre 1987

La fertilisation terminologique dans les langues romanes

URI : https://id.erudit.org/iderudit/002272ar

DOI : https://doi.org/10.7202/002272ar

Aller au sommaire du numéro

Éditeur(s)

Les Presses de l'Université de Montréal

ISSN

0026-0452 (imprimé)

1492-1421 (numérique)

Découvrir la revue

Citer cet article

Clas, A. (1987). Une matrice terminologique universelle : la brachygraphie gigogne. Meta, 32(3), 347-355. https://doi.org/10.7202/002272ar
Résumé de l'article

La brachygraphie gigogne, c'est-à-dire la formation des divers types de mots valises, est un procédé de formation de mots nouveaux, une matrice terminologique. L'étude analyse les procédés formels et syntaxico-sémantiques et donne de nombreux exemples en français, anglais, espagnol et italien. L'intérêt du procédé dans les études psychopathologiques et psychanalytiques est aussi évoqué. 


\section{UNE MATRICE TERMINOLOGIQUE UNIVERSELLE : LA BRACHYGRAPHIE GIGOGNE}

ANDRÉ CLAS

Université de Montréal, Montréal, Canada

\section{RÉSUMÉ}

La brachygraphie gigogne, c'est-à-dire la formation des divers types de mots valises, est un procédé de formation de mots nouveaux, une matrice terminologique. L'étude analyse les procédés formels et syntaxico-sémantiques et donne de nombreux exemples en français, anglais, espagnol et italien. L'intérêt du procédé dans les études psychopathologiques et psychanalytiques est aussi évoqué.

\section{INTRODUCTION}

La brachygraphie a déjà fait couler beaucoup d'encre, comme on peut le voir dans la bibliographie citée. Si nous nous permettons de revenir sur un aspect particulier de la brachygraphie, c'est-à-dire sur la brachygraphie que nous appelons gigogne ou, si l'on préfère, sur la formation de mots valises, de portemanteaux words comme on dirait en anglais, c'est parce que nous croyons y trouver un modèle de formation dont aucune langue ne peut se passer, tout simplement parce qu'il s'agit d'un procédé linguistique économique - et en plus quelque peu ludique, ce qui n'est pas un défaut, mais bien au contraire, une garantie de rétention et de diffusion - bref, c'est parce que nous croyons qu'il y a dans ce procédé une matrice terminologique, universelle qui peut répondre à des besoins de créativité. Nous nous proposons donc d'examiner les modèles formels et les modèles sémantiques que l'on peut dégager, de formuler des remarques pertinentes et d'examiner les " réactions " en suivant les mêmes concepts exprimés linguistiquement dans d'autres langues.

\section{LES MODÈLES FORMELS}

Devant la diversité des dénominations des mêmes processus d'abrègement, nous avons décidé d'intituler notre étude brachygraphie gigogne pour montrer qu'il s'agit d'écritures tronquées qui s'emboîtent et pour éviter les termes plus ou moins marqués tels que hapaxépie, haplolagie, haplologie, acronymie, crase, paronomase, croisement, amalgame, télescopage, emboîtement, ou encore mot valise, mot centaure, mot gigogne, mot contaminé, mot fusionné, mot portemanteau. Remarquons que la même richesse synonymique existe en anglais où l'on parle de blends, de blend-words, de blending, name-fusion, de portemanteau-words; les Allemands parlent de Wortverschmelzung, de Portemanteau-Wörter; les Italiens intitulent le procédé de façon fort poétique "parole macedonia ».

Mais voyons les modèles formels. La brachygraphie gigogne repose fondamentalement sur l'utilisation combinée de trois procédés, à savoir l'apocope, l'aphérèse et la syncope. La combinaison des procédés donnent en principe les modèles théorique suivants : 
Modèle 1 : apocope et aphérèse

Modèle 2 : apocope et apocope

Modèle 3 : aphérèse et aphérèse

Modèle 4 : apocope simple

Modèle 5 : aphérèse simple

Modèle 6 : apocope et syncope

Si théoriquement tous les modèles sont possibles, il faut cependant faire remarquer que la productivité des modèles est variable et que le modèle canonique semble bien être le premier, qui est le plus productif. Voyons quelques exemples :

1.1 Modèle 1 (apocope et aphérèse) :

$\begin{array}{ll}\text { stagflation } & \text { stagnation + inflation } \\ \text { télérendum } & \text { télévision + référendum } \\ \text { cubitaine(u)r } & \text { cubique + containe(u)r } \\ \text { restoroute (restauroute) } & \text { restaurant + autoroute } \\ \text { plapier } & \text { plastic + papier } \\ \text { altiport } & \text { altitude + aéroport } \\ \text { chambrion } & \text { chambre + ionisation } \\ \text { escool } & \text { essence + alcool } \\ \text { carburol } & \text { carburant + alcool } \\ \text { mécanotrique } & \text { mécanique + électronique } \\ \text { cybernation } & \text { cybernétique + automation } \\ \text { cardulance } & \text { cardiologie + ambulance } \\ \text { progiciel } & \text { programme + logiciel } \\ \text { bionique } & \text { biologie + électronique } \\ \text { platformage } & \text { platinium + reforming (age) } \\ \text { castode } & \text { cascade + triode } \\ \text { cyclotron } & \text { cycle + électron } \\ \text { magnétron } & \text { magnéto + cyclotron } \\ \text { gélule } & \text { gélatine + capsule } \\ \text { transistor } & \text { transfer + resistor } \\ \text { camescope } & \text { caméra + magnétoscope }\end{array}$

Mais arrêtons là pour ne pas allonger indûment la liste. On voit immédiatement que le modèle 1 est très productif. On voit aussi que certains exemples ne sont pas tout à fait des formations tirées directement du français puisque leur concaténation originelle n'est pas celle du français (plapier, cubitaineur). D'une certaine façon, on peut les traiter comme des emprunts. On peut encore faire remarquer que certaines formations ont été créées après une analyse du mot valise en anglais. On a ainsi camescope : caméra + magnétoscope (modèle avec apocope, aphérèrse et syncope), c'est-à-dire caméra électronique avec magnétoscope intégré, où le point de départ est bien camcorder : camera + recorder. Il y a donc là un bel exemple de fertilisation terminologique. Il faut encore signaler que les formations s'intègrent à la langue et peuvent donc former des dérivés normaux. Ainsi mécatronique, c'est-à-dire l'électronisation de la mécanique, a un dérivé mécatronicien, le spécialiste de la mécatronique. On constate encore que l'on a une série formée avec ce que l'on peut de plus en plus considérer comme un suffixe, soit donc la série formée avec -ique. La terminaison -ique est polysème et condense à la fois le signifié informatique, le signifié automatique ou le signifié électronique. Il est vrai qu'il y a certaines relations entre les trois signifiés et que l'ambiguïté ne semble pas trop gênante. 
On a ainsi :

$\begin{array}{ll}\text { mécatronique } & \text { mécanique + électronique } \\ \text { domotique } & \text { domicile + électronique } \\ \text { transitique (modèle 5) } & \text { transit + automatique } \\ \text { usinique } & \text { usine }+ \text { électronique } \\ \text { télématique } & \text { télétransmission }+ \text { informatique } \\ \text { avionique } & \text { avion }+ \text { électronique } \\ \text { robotique (modèle 5) } & \text { robot }+ \text { informatique } \\ \text { bureautique (modèle 5) } & \text { bureau + informatique } \\ \text { intellectique } & \text { intelligence }+ \text { informatique } \\ \text { terminotique } & \text { terminologie + informatique } \\ \text { créatique } & \text { création }+ \text { informatique }\end{array}$

Il peut aussi y avoir une double formation comme dans tangélo : tangerine + pomélo, où pomélo vient de pomum + melo.

1.2 Modèle 2 (apocope + apocope)

\begin{tabular}{|c|c|}
\hline chloroforme & chlore + formyle \\
\hline aldéhyde & alcool + déhydrogenatum \\
\hline cermet & ceramique + metal \\
\hline telex & teleprinter + exchange \\
\hline modem & modulateur + démodulateur \\
\hline sial & silicium + aluminium \\
\hline syndet & synthétique + détergent \\
\hline pixel & picture + element \\
\hline chambrion & chambre + ionisation \\
\hline héliox & hélium + oxygène \\
\hline
\end{tabular}

On peut faire remarquer ici que le modèle ne semble pas, à part les exemples de composés chimiques, être aussi productif et que la majorité des formations peut être classée comme emprunt. Le modèle existe pourtant en français puisque maillechort date de 1829 et est formé des noms des inventeurs, Maillot et Chorier (l'alliage de cuivre, de zinc et de nickel qui imite l'argent). Une formation plus récente tachèle : tache et élémentaire (télédétection). Peut-être faut-il faire entrer ici la formation napalm : $\mathrm{Na}$, symbole du sodium et palmitate?

\subsection{Modèle 3 (aphérèse et aphérèse)}

Les exemples sont beaucoup plus rares et la seule attestation que nous ayons, pour le moment, est nylon : vinyl + coton. Encore s'agit-il d'un emprunt.

\subsection{Modèle 4 (apocope simple)}

$\begin{array}{ll}\text { distribanque } & \text { distributeur + banque } \\ \text { zircuivre } & \text { zirconium + cuivre } \\ \text { zircalloy } & \text { zirconium + alloy (alliage) } \\ \text { publipostage } & \text { publicité + postage } \\ \text { infographie } & \text { informatique + graphie } \\ \text { gyropilote } & \text { gyroscope + pilote } \\ \text { caloporteur } & \text { calorifere + porteur } \\ \text { mouchèvre } & \text { mouton + chèvre }\end{array}$

Le modèle conserve intact le deuxième élément. Ce modèle ne semble pas récent puisqu'on peut relever les exemples crapoussin : crapeau et poussin, c'est-à-dire " personne petite et trapue ", daté de 1752 . La création permalloy : perméable + alloy, date 
de 1923 et permafrost : permanent + frost, date de 1946 . À cette série s'ajoute une formation plus compliquée pergélisol : permanent + gel + sol, qui date de 1946, ou encore prostaglandine : prostate + glande + ine, qui date de 1936, même type de formation que l'on trouve dans bacitracine : bacille + trace + ine (mot anglais : du latin bacillum et de l'anglais trace), Faut-il ajouter à la série manouvrier : manœuvre + ouvrier, qui date de 1870, mais que le Petit Robert fait remonter à 1189, au latin médiéval, manuoperarius!

$\begin{array}{cl}1.5 \text { Modèle } 5 \text { (aphérèse simple) } & \\ \text { bureautique } & \text { bureau + informatique } \\ \text { robotique } & \text { robot + informatique } \\ \text { avionique } & \text { avion + électronique } \\ \text { télématique } & \text { télétransmission }+ \text { informatique } \\ \text { fibromyome } & \text { fibrome + myome } \\ \text { foultitude } & \text { foulte et multitude } \\ \text { tigron } & \text { tigre + lion }\end{array}$

Là encore le modèle n'est pas entièrement récent puisque foultitude remonte à 1848 et que tigron (ou tiglon) est daté de 1937. Dans la même série, on peut encore ranger les formations lombricompost : lombric + compost et lombricompostage. Le modèle est productif puisque quelqu'un a récemment proposé fourmandises pour traduire bake sale! Remarquons en passant qu'on trouve dans les dictionnaires courants : bistroquet (1926) : bistro + troquet, bisbrouille : bisbille et brouille, bitumastic : bitume + mastic.

\subsection{Modèle 6 (apocope ou aphérèse et syncope)}

Ce modèle est très hybride et les exemples peuvent parfois apparaître comme relevant de la siglaison ou de l'acronymie. On a ainsi :

$\begin{array}{ll}\text { upérisation } & \text { ultra + pasteurisation } \\ \text { amatol } & \text { ammonium nitrate + trinitoluène } \\ \text { carburanes } & \text { carbone, hydrocarbures + uranium } \\ \text { limonette } & \text { limette + lemon } \\ \text { paddair } & \text { paddle tennis plein air }\end{array}$

Peut-être peut-on ranger dans ce type des formations comme secam : séquentiel à mémoire, qui relève tout aussi bien d'un autre modèle de brachygraphie, l'acronymie, ou encore franglais, formé soit de fr + anglais ou de fran + glais et relevant alors du modèle 1 .

\section{CONSIDÉRATIONS PHONÉTIQUES}

Si l'on examine les divers exemples de brachygraphie gigogne, on constate qu'il se produit diverses réactions. Le cas le plus simple, et c'est certainement pour cela qu'on parle de croisement ou d'amalgame, est celui où deux syllabes ou deux phonèmes sont identiques dans les deux unités de formation et se fondent, c'est le phénomène de l'hapaxépie ou haplologie. Exemples : français + anglais : franglais ; lombric + compost : lombricompost ; four + gourmandises : fourmandises ; foul + multitude : foultitude ; stagnation + inflation : stagflation. Les auteurs comme J. Laforgue, Michaux, Queneau, Céline, Daudet, Ionesco, Audiberti, sans oublier San Antonio se servent abondamment de ce procédé ludique pour créer des jeux de mots où la transparence est presque automatique. On en trouvera une longue liste dans le Dictionnaire des mots sauvages. Les autres variations sont des adaptations au système phonologique ou graphique de la langue. Par exemple : secam : q $\longleftrightarrow$ c, tachèle $:$ é $\longleftrightarrow$ è, en syllabe fermée. 


\section{ASPECTS SYNTAXICO-SÉMANTIQUES}

Si on examine les aspects syntaxico-sémantiques des formations brachygraphiques gigognes, dans lesquelles les deux composants $\mathrm{A}$ et $\mathrm{B}$ donnent le résultat $\mathrm{C}$, on peut dégager les deux types suivants :

3.1 Le résultat $\mathrm{C}$ est un $\mathrm{A}$ et un $\mathrm{B}$. Il y a formation d'un nouveau signifié, c'est-à-dire il y a création d'un dvandva. La stagflation est à la fois la stagnation et l'inflation, c'est l'un et l'autre. L'escool est un mélange d'essence et d'alcool, la camescope une caméra et un magnétoscope. On peut regrouper dans cette catégorie la majeure partie des nombreuses formations de composés chimiques (chloroforme, nylon, ...) et des croisements biologiques (tigron, mouchèvre, pomate, tangélo, ...).

3.2 Le résultat $C$ est modifié par A ou par B. Ainsi, un progiciel est un B (logiciel) avec un $A$ (programme : un programme de logiciel), plapier est un $B$ (papier) en $A$ (plastique), un cubitaineur est un B (container) qui est comme un A (cubique), le publipostage est un B (publicité) faite par B (postage), tout comme l'infographe est un B (graphie) faite à l'aide de $\mathrm{A}$ (informatique). On voit que la décomposition sémantique peut être variée, il y a même plusieurs possibilités pour une même formation, mais l'ensemble peut être regroupé sous l'étiquette formations endocentriques. L'analyse syntaxicosémantique montre bien que la brachygraphie gigogne n'est au fond qu'un cas particulier de la composition. Comme le souligne Jean Tournier (1985:30) : "Morphologiquement, la seule différence est que les éléments de l'amalgame sont plus ou moins emboités les uns dans les autres ou, comme on dit 'télescopés', alors qu'ils sont juxtaposés dans les autres composés."

On pourrait ici reprendre de façon détaillée les diverses analyses syntaxiques proposées pour le $2^{\mathrm{e}}$ type de brachygraphie gigogne et répartir les exemples en suivant l'analyse de Adams, citée par Jean Tournier (1985: 132). On aurait ainsi :

a) Un prédicat nominal : ambucopter : the ambulance is a helicopter.

b) Un adjectival :

1) avec un adjectif medicare : the care is medical;

2) avec un adjectif dérivé : musicomedy : the comedy is musical;

3) avec un participe passé : spam : the ham is spiced.

c) Sujet et objet

1) But : a) objet — nom : motel : the motel is for motorist ; b) objet - nominalisation : navicert : the certificate is for navigation; c) prépositionnel : chunnel : the tunnel is under the channel.

2) Sujet et verbe objet : a) ordre 1.2 : Warphan : the war causes the orphan; b) ordre 2.1 : opinionaire : the questionnaire elicits opinions; c) 2 contient/est formé de 1 : motorcade : the calvacade consists of motor cars.

d) Verbe et objet

1) verbe $\longrightarrow$ nom d'agent : breathalyser : (the device) analyses breath

2) verbe $\longrightarrow$ nominalisation : colourcast : it is broadcast in colour.

e) Divers : screamager (screaming teenager), fruice (fruit juice).

Cette analyse est plausible, mais elle laisse cependant place à d'autres interprétations et, au fond, ne fait que "souligner la variété des manifestations du processus " (Tournier 1985 : 133). Que nous apportent au fond des analyses du type suivant :

$\begin{array}{ll}\text { restauroute } & \text { le restaurant est près de l'autoroute } \\ \text { plapier } & \text { le papier est en plastique } \\ \text { cubitainer } & \text { le containeur est cubique } \\ \text { terminotique } & \text { la terminologie utilise l'informatique ? }\end{array}$


La décomposition sémantique n'est pas toujours très convaincante. Ainsi, comment analyser progiciel ? Le programme est de logiciels ? Ou, les logiciels sont un programme?

Quoi qu'il en soit, nous devons retenir que la brachygraphie est un procédé bien vivant et qu'on retrouve dans beaucoup de langues. Il y a là, à la fois, un procédé de création authentique pour chaque langue et en même temps un procédé qui permet l'absorption facile d'emprunts. La "fertilisation " est donc bien garantie!

\section{EXEMPLES DANS D'AUTRES LANGUES ROMANES}

Nous avons grâce à Mme Christina Allegra, terminologue italienne à Bruxelles et à M. Fabrizio Megale, traducteur à Rome, pu constituer une petite liste de "parole macedonia " en italien, et nos collègues à Montréal nous ont signalé quelques exemples en espagnol. Qu'ils en trouvent ici notre gratitude.

\subsection{La brachygraphie gigogne en italien}

Voyons quelques exemples :

\section{PAROLE MACEDONIA}

\section{- fatturatica}

(da : fatturazione + informatica)

DF : Tecnica dei sistemi di fatturazione, da eseguirsi mediante macchine contabili elettroniche computerizzate. (Devoto-Oli, supplemento)

agronica

(da : agronoomia + elettronica)

DF : disciplina volta ad applicare tecnologie e prodotti elettronici all'agricoltura $e$ alla zootecnica per renderne pi $\dot{J}$ agevole e redditizia la produzione. (Nuovo Zingarelli)

- altiporto o altoporto)

(da : altopiano + aeroporto)

DF : Aeroporto di montagna. (Devoto-Oli, supplemento)

\section{autoplano}

(da : automobile + aeroplano)

DF : mezzo di trasporto costituito dall'unione di un'automobile con un piccolo aeroplano. (Devoto-Oli, supplemento)

\section{agriplano}

\section{macchina agricola volante.} (Mondo economico, $\mathrm{n}^{\circ} 25,6-1985, \mathrm{p} .55$.)

(da : " abitare» + "container 》)

\section{abitainer}

no 48,15 dic. 1982 , p. 81 .)

turismatica

mecatronico controllo e azionamento.
monetica
(da : monetario + informatica)
eidomatica
informatica delle immagini

DF : creazione e elaborazione di immagini e figure mediante computer. (Bibliot. italiana) 
- carbodotto

- elicostato

DF : nuovo apparecchio studiato per $i$ tagli di foresta, fungente di volta in volta da elicottero e da pallone aerostatico. (Bibliot. italiana)

fluidica

DF : tecnica delle logiche a fluido. (Bibliot. italiana)

- ludotica

DF : giochi sul calcolatore. (Bibliot. italiana) (da « carbone»+ " condotto $)$

(elicottero + aerostato)

(da : "fluido»+ + logica ")

\section{mandarancio}

$$
\text { (da : triticum + segale) }
$$

(Fr. : clémentine)

\section{- stagfiazione \\ (da : stagnazione + inflazione)}

DF : concomitanza dei due fenomeni economici di stagnazione (recessione) e d'inflazione. (Devoto-Oli, supplemento)

colf

bidonvia

DF : Impianto di salita e discesa per sciatori, simile alla seggiovia, ma con piccole cabine scoperte a forma cilindrica, per due persone, al posto dei sedili. (Devoto-Oli, supplemento)

\section{- bionica}

DF : studio della struttura e delle funzioni degli organismi viventi, allo scopo di trarne elementi utili per invenzioni o innovazioni tecnologiche. (Devoto-Oli, supplemento)

- elibus

(da : elicottero + autobus)

DF : elicottero attrezzato e impiegato per il trasporto pubblico di persone. (DevotoOli, supplemento)

\section{filanca}

(da : filato + Rumianca, nome della società chimica produttrice della fibra)

DF : nome commerciale depositato di una fibra tessile sintetica dodata di grande elasticitaj.

- pulsar (dall'inglese : pulsating + radio source) DF : denominazione di radiosorgenti che emettono periodicamente radioconde sotto forma di brevi impulsi. (Devoto-Oli, supplemento)

Si on examine quelques exemples, on voit que le procédé brachygraphique est bien le même et qu'il y a des formations autochtones, des formations empruntées adaptées et 
des formations empruntées telles quelles. On a ainsi : anglais : stagflation, français : stagflation, italien : stagflazione, et d'un autre côté, napalm, modem, sial, etc. en italien.

La longue liste d'exemples montre bien que ce procédé existe de façon très vivante

\subsection{La brachygraphie gigogne en espagnol}

L'espagnol connaît les mêmes types de formation. La langue usuelle fabrique facilement des formations comme narigudo, "grand nez, nez pointu ", avec nariz " nez " et agudo " aigu ", ou encore : barrigudo de barriga, "neutre " et agudo, "prononcé "; naguldo, nalga «fess " et agudo, "prononcée ". Dans le domaine du vocabulaire spécialisé, l'espagnol a adapté bionica, biotica, aldebrido, robotica, telematica, chloroformo et a emprunté les formations napalm, transistor.

On voit donc là aussi que le procédé est tout à fait vivant et productif. En fait, le procédé est tellement vivant qu'il intéresse non seulement les poètes et les littérateurs, mais aussi les psychanalystes, les psychologues et les aphasiologues.

\section{REMARQUES PSYCHOPATHOLOGIQUES}

Nous n'avons nullement l'intention de nous lancer dans tous ces domaines et nous ne voulons que signaler le phénomène pour bien montrer qu'il y a là un domaine de recherche universel. C'est ainsi, à titre d'illustration, on trouvera les références dans la bibliographie, que le Dr Lecours et ses collaborateurs distinguent quatre types de " télescopages " provenant de locuteurs normaux, aphasiques et schizophases.

- Le télescopage phonémique : unité empruntant ses phonèmes à au moins deux mots cibles; respecte le système phonologique : mammifère + animal $\longrightarrow$ mamimal.

- La paraphasie monémique dérivée : unité empruntant ses monèmes à au moins deux mots cibles; respecte le système morphologique : allumer + fumer + cigarette $\longrightarrow$ afumette.

- Le télescopage verbal : l'unité respecte le système phonologique et morphologique : série de symptômes + kyrielle de symptômes sériel de symptômes.

- Le télescopage syntagmatique : l'unité emprunte ses mots à au moins deux syntagmes cibles, reliés sur le plan conceptuel et/ou formel : le plus possible + le plus que je veux $\longrightarrow$ le plus que je possible.

\section{CONCLUSION}

La brachygraphie gigogne fait, comme nous l'avons vu, partie de notre univers. C'est un procédé de formation de mots qui présente beaucoup d'avantages et qui est universel. Peut-être faut-il ici signaler qu'il se pourrait qu'une des premières formations brachygraphiques gigognes du français soit le mot haut. En effet, haut est peut-être formé sur le francique hoch et le latin altus? Pour bien montrer la fécondité du processus, rappelons pour terminer la formation d'autobus et la richesse de dérivation qui en découle. Autobus a été formé en 1907, d'après le Petit Robert, par brachygraphie gigogne avec automobile et omnibus. Depuis, la réduction bus est devenue un suffixe comme le prouvent les formations : airbus, aérobus, cinébus, bibliobus, muséobus, ...

\section{BIBLIOGRAPHIE}

ALGEO, J. (1977) : "Blends, a Structural and Systematic View", American Speech 52, pp. 47-64.

BAUER, L. (1983) : English Word-Formation, Cambridge, Cambridge University Press, pp. 234-240.

BOVREL, A. \& J.-L. NESPOULOUS (1977) : "A Psycho-Linguistic Analysis of Several Substitution Phenomena in Paraphrastic Speech", International Journal of Psycholinguistics, 1, pp. 13-30.

BUCKINGHAM, H.W. Jr. \& A. KERTESA (1974) : " A Linguistic Analysis of Fluent Aphasia ", Brain \& Language, 1, pp. 43-62. 
BUCKINGHAM, H.W. Jr., H. AVAKIAN-WHITAKER \& H.A. WHITAKER (1978) : "Alliteration and Assonance in Neologistic Jargon Aphasia ", Cortex, XIV, pp. 365-380.

BUCKINGHAM, H.W. Jr. (1979) : " Linguistic Aspects of Lexical Retrieval Disturbances in the Posterior Fluent Aphasics ", in : H. Whitaker \& H.A. Whitaker (Eds.), Studies in Neurolinguistics, vol. 4, New York, Academic Press, pp. 269-291.

BUCKINGHAM, H.W. Jr., H. WHITAKER \& H.A. WHITAKER (1979) : "On Linguistic Perseveration ", in : H. Whitaker \& H.A. Whitaker (Eds.), Studies in Neurolinguistics, vol. 4, New York, Academic Press, pp. 330-352.

BUCKINGHAM, H.W. Jr. (1980) : "On Correlating Aphasic Errors with Slips-of-the-Tongue ", Applied Psycholinguistics, 1, pp. 199-220.

BUCKINGHAM, H.W. Jr. (1980) : "Where Do Neologisms Come From? ", in J. Brown (Ed.), Jargonaphasia, New York, Academic Press, pp. 39-62.

CHAURAND, J. (1977) : "Des croisements aux mots-valises ", le Français moderne, xICv, pp. 4-15.

CLAS, A. (1984) : "Brachyphonie et brachygraphie", Lebende Sprachen, Heft 3, xxix, Jahrgang, pp. 118-119.

CLAS, A. (1985) : "Composés lourds et créations brachygraphiques", la Banque des mots, vol. 30, pp. $135-145$.

DIETRICKS, J. (1966) : "Les mots-valises de l'anglais et du français", Revue des langues vivantes 32, pp. 451-459.

FINKIELKRAUT, A. (1979) : Ralentir : mots-valises, Paris, Seuil.

FINKIELKRAUT, A. (1981) : Petit dictionnaire illustré, Paris, Seuil.

FORGUE, G.J. et R.I. McDAVID (Jr) (1972) : la Langue des Américains, Paris, Aubjer, pp. 153-154.

GILBERT, P. (1971) : Dictionnaire des mots nouveaux, Paris, Hachette-Tchou.

GRÉSILLON, A. (1983) : "Mi-figue mi-raisin. Dévaliser des mots-valises", $D R L A V, \mathrm{n}^{\circ} 24$, pp. 83-107

GRÉSILLON, A. (1984) : la Règle et le monstre : le mot-valise, Tübingen, Niemeyer.

GUILBERT, C. (1975) : la Créativité lexicale, Paris, Larousse.

GUIRAUD, J., P. PAMART et J. RIVERAIN (1971) : les Mots dans le vent, Paris, Larousse.

GUIRAUD, J., P. PAMART et J. RIVERAIN (1974) : les Nouveaux Mots dans le vent, Paris, Larousse.

LECOURS, A.R. (1975) : " Methods for the Description of Aphasic Transformation of Language ", in : E.H. Lenneberg \& E. Lenneberg (Eds.), Foundations of Language Development, vol. 2, New York, Academic Press, pp. 75-94.

LECOURS, A.R. \& F. ROULLLON (1976) : "Neurolinguistic Analysis of Jargonaphasia and Jargonagraphia ", in : H. Avakian-Whitaker \& H. Whitaker (Eds.), Studies in Neurolinguistics, vol. 2, New York, Academic Press, pp. 95-144.

LECOURS, A.R. \& M. VANIER-CLÉMENT (1976) : "Schizophasia and Jargonaphasia ", Brain \& Language, 3, pp. 516-565.

LECOURS, A.R. \& F. LHERMITTE (1979) : l'Aphasie, Paris \& Montréal, Flammarion Médecine-Sciences \& Les Presses de l'Université de Montréal.

LECOURS, A.R. (1982) : "On Neologisms", in : J. Mehler, E.C.T. Walker \& M.F. Garrett (Eds.), Perspectives on Mental Representation, Hillsdale, Erlbaum, pp. 217-247.

LECOURS, A.R., E. OSBORN, L. TRAVIS, F. ROUILLON \& G. LAVALLÉE-HUYNH (1981) : "Jargons", in : J. Brown (Ed.), Jargonaphasia, New York, Academic Press, pp. 9-38.

LECOURS, A.R., M. NAVET \& A. ROSS-CHOUINARD (1981) : "Langage et pensée du schizophase", Confrontations psychiatriques 19, pp. 109-144.

MARCHAND, H. (1969) : The Categories and Types of Present-Day English Word Formations, München, Beck.

NESPOULOUS, J.-L. et A.R. LECOURS (1980) : " Du trait au discours : les différents niveaux de structuration du langage et leur atteinte chez les aphasiques", Grammatica VII, no 1, Toulouse, Université de Toulouse-Le Mirail, pp. 1-36.

RHEIMS, M. (1969) : le Dictionnaire des mots sauvages, Paris, Larousse.

SAUVAGEOT, A. (1964) : Portrait du vocabulaire français, Paris, Larousse.

SCHWARTZ, U. (1970) : "Die Strukkur der englischen Portemanteau-Words", Linguistische Berichte, no 7 , Braunschweig, Vieweg, pp. 40-44.

TOURNIER, J. (1985) : Introduction descriptive à la lexicogénétique de l'anglais contemporain, ParisGenève, Slatkine, pp. 130-138. 